Revue d'histoire de l'Amérique française

RAS REVUE D.HISTOIRE DE L'AMÉRIQUE FRANÇAISE

\title{
Charles d'Aulnay et la Compagnie de la Nouvelle-France
}

\section{René Baudry}

Volume 11, numéro 2, septembre 1957

URI : https://id.erudit.org/iderudit/301833ar

DOI : https://doi.org/10.7202/301833ar

Aller au sommaire du numéro

Éditeur(s)

Institut d'histoire de l'Amérique française

ISSN

0035-2357 (imprimé)

1492-1383 (numérique)

Découvrir la revue

Citer cet article

Baudry, R. (1957). Charles d'Aulnay et la Compagnie de la Nouvelle-France. Revue d'histoire de l'Amérique française, 11(2), 218-241.

https://doi.org/10.7202/301833ar d'utilisation que vous pouvez consulter en ligne.

https://apropos.erudit.org/fr/usagers/politique-dutilisation/ 


\section{CHARLES D'AULNAY}

\section{ET LA}

\section{COMPAGNIE DE LA NOUVELLE-FRANCE}

Les musées et les panthéons exigent, comme les maisons et les rues, des nettoyages périodiques. Dieu sait combien de faux chefs-d'œuvre, de bustes en toc et de pseudo-héros, on vénère parfois dans ces temples de la gloire! Le musée imaginaire de l'histoire a, lui aussi, besoin périodiquement d'aération et de rajeunissement. Pour répondre sans doute à ce besoin d'hygiène, nos historiens canadiens éprouvent, de temps en temps, des fureurs d'iconoclastes, et partent en guerre contre les idoles élevées par leurs devanciers. La plupart de nos grands hommes, Champlain, Dollard, Frontenac, ont servi de cibles à ce jeu de massacre. Cet amusement a causé un peu de tapage, et troublé nombre de bonnes gens dans leurs admirations patriotiques. Faut-il vraiment s'en alarmer ? Pourvu qu'agresseurs et défenseurs observent les règles d'une critique loyale, leurs discussions servent à assainir et vivifier l'histoire. Le pis qui puisse arriver serait de dégonfler quelques réputations boursouflées, ou de démolir quelques statues de plâtre. Les monuments et les réputations solides résisteront bien aux attaques. Ces divergences de vues et les mouvements de hausse et de baisse, à la bourse des valeurs historiques, peuvent aussi nous montrer la valeur précaire de nos jugements, et nous inviter à plus d'objectivité.

L'un des principaux personnages dans les annales de l'Acadie, Charles de Menou, sieur d'Aulnay, a déjà connu des fortunes très diverses. D'abord jugé sévèrement par Denys, Charlevoix et Garneau, il a joui d'un regain d'estime, depuis qu'en 1873 M. Charles Moreau ${ }^{1}$ a entrepris de le réhabiliter, suivi plus tard

1 Dans son Histoire de l'Acadie françoise, publiée d'abord par tranches dans Le Cabinet historique, vol. 13 à 18 (1867 à 1872), puis en volume à petit tirage. 
avec enthousiasme par M. Émile Lauvrière. L'excès de faveur dont il a bénéficié depuis, au détriment de ses adversaires, a même provoqué une vive polémique, où des historiens ecclésiastiques et laïcs se sont lancé de gros livres à la tête. ${ }^{2}$ Cette rivalité posthume a provoqué des recherches passionnées, et les auteurs actuels peuvent formuler un verdict plus pondéré et plus juste.

Mais il est bien difficile d'écrire l'histoire définitive. Comme tout art et toute science vivante, l'histoire refuse de se laisser fossiliser. Elle ne peut demeurer statique, mais cherche sans cesse à progresser, à mesure que de nouveaux renseignements voient le jour, ou que s'élaborent des interprétations plus exactes. Le débat sur d'Aulnay vient à peine de se clore, qu'un nouveau document nous invite à le reprendre. Comment voulez-vous donc que les professeurs et les auteurs de manuels trouvent le temps d'apprendre leur histoire, de même que leur géographie, puisqu'elles changent toujours !

Cette pièce, qui eût causé une joie extrême à l'abbé CouillardDesprés, se trouve à la Bibliothèque Mazarine. ${ }^{3}$ Elle se présente sous la forme d'un feuillet imprimé, de 8 pages in-4, et porte le titre de Factum pour les Directeurs et Associez de la Compagnie de la Nouvelle-France, demandeurs et complaignants, contre le Sieur d'Aunay, deffendeur et accusé. C'est un réquisitoire présenté à la Cour, pour demander une information judiciaire contre d'Aulnay, et sa révocation comme gouverneur. Nous avions déjà vu ce personnage en rivalité avec beaucoup de monde: avec Denys, Latour et autres. Cette pièce nous le montre cette fois en opposition violente, lui, lieutenant-général du Roi en Acadie, avec la puissante Compagnie des Cent Associés, suzeraine du pays. Ce conflit, dont on ne trouve guère d'autre trace, atteignit son point culminant en 1647 , mais il couvait, semble-t-il, depuis

2 Cette querelle de famille a produit une bonne dizaine d'ouvrages. En faveur d'Aulnay intervinrent Lauvrière et le R.P. Candide de Nant; pour Latour, l'abbé Couillard-Després et M. Leander d'Entremont.

3 Recueil A.15,444, No 33 . Cette bibliothèque, peu explorée, possède plusieurs pièces anciennes, intéressantes pour le Canada. Cet exemple et quelques autres font penser qu'une prospection méthodique dans les bibliothèques de France, comme celle qu'a faite G. Atkinson sur La Littérature géographique française de la Renaissance, apporterait un bon nombre d'indications nouvelles à la bibliographie et à l'histoire canadiennes. 
au moins une dizaine d'années. Pour comprendre le fond de cette affaire, il est nécessaire de la replacer dans son contexte de circonstances.

Rappelons-nous tout d'abord que deux adversaires de Charles d'Aulnay entretinrent des relations étroites avec la Compagnie de Nouvelle-France. Nicolas Denys, marchand à La Rochelle, recrutait pour elle des engagés et organisait des armements. Il en avait obtenu des commissions pour Miscou et le Cap-Breton. Un autre pionnier acadien, Charles de Latour, avait aussi déjà reçu des secours des Associés. Nommé par la compagnie lieutenant-général en 1631, il se dit « ung des associés d'icelle », dans l'acceptation de ses lettres-patentes. Les Latour avaient déjà fondé un poste à Pentagouët, dont les Anglais s'emparèrent. Quand d'Aulnay voulut reprendre cette place, Charles de Latour se trouva lésé, et refusa de collaborer. La Compagnie prit le parti des Latour, en 1636, en concédant ce poste à Claude de Latour. Mais le Roi intervint à son tour, pour attribuer, peu après, cette place à d'Aulnay. "L'invictualement de Pentagouet » 4 fut donc l'amorce lointaine d'une longue rivalité, non seulement entre d'Aulnay et Latour, mais aussi entre d'Aulnay et la Compagnie.

Rappelons encore que d'Aulnay n'obtint aucune charge directement de la Compagnie, sauf le commandement d'un vaisseau, en 1633, sur la recommandation du commandeur de Razillly. Il tint ensuite ses pouvoirs exclusivement des Razilly et du Roi. Quand il acheta les parts de Claude de Razilly, en 1642, la Compagnie enregistra bien cette transaction, mais négligea ou refusa de le présenter comme gouverneur. D'Aulnay s'adressa donc directement à la Cour, pour obtenir la confirmation de ses pouvoirs. Dès qu'il l'eut obtenue de façon catégorique, en 1647, il entreprit de confisquer les forts de ses concurrents, concessionnaires de la Compagnie. Devant cette attaque directe à leurs droits et à leurs biens, les Associés qui, jusque là, n'avaient offert qu'une assez molle opposition aux empiètements d'Aulnay, réagirent en présentant contre lui une lourde charge à la Cour.

4 Arrêt du Conseil privé, 1er février 1641. Copie de Margry à la B.N., N.A.F. 9281:84. 
Ce Factum relate les événements de 1627 à 1647, et couvre donc les vingt premières années de la seconde colonisation acadienne. Comme les documents sur cette époque n'abondent guère, nous croyons utile d'analyser celui-ci en détail. Nous devons cependant ne pas oublier qu'il s'agit d'un plaidoyer, c'est-à-dire d'une pièce intéressée, qui n'expose qu'un aspect de la réalité. Il faudra donc la confronter soigneusement avec les autres documents de l'époque, et contrôler ses affirmations, afin d'en dégager les données certaines.

La première partie du Factum forme un préambule, et nous rapporte des faits connus: la création de la Compagnie en 1627, ses privilèges, son premier envoi de six vaisseaux à Québec, intercepté par les Kirke, et la reprise, par le capitaine Daniel, de quelques postes au Cap-Breton et à Miscou.

On peut noter, au passage, que l'acte de création de la Compagnie de Nouvelle-France ne mentionnait pas nommément l'Acadie. Ni Richelieu, ni les principaux associés ne semblaient savoir qu'un petit groupe des colons de Poutrincourt continuait d'habiter la péninsule. Seuls quelques marchands de Bordeaux et de La Rochelle gardaient relations avec eux. Les lettres de Latour au Roi et à Richelieu, la tentative d'établissement des Écossais à Port-Royal et au Cap-Breton, le récit du capitaine Daniel, et surtout les représentations de Champlain, attirèrent l'attention du ministre et des Associés de ce côté. Richelieu, dans les délibérations du traité de Saint-Germain-en-Laye, réclama la restitution de Port-Royal et de Pentagouët, et dès 1630 quelques associés de Bordeaux envoyèrent des renforts à Latour. ${ }^{5}$ Mais le Factum ne fait aucune mention de ces armements et ne parle aucunement de Latour, pour une raison évidente. Depuis 1645 , ce dernier était en complète disgrâce et déconfiture. On le considérait comme un traître; il avait été condamné et ses forts,

5 Sur les activités de la Compagnie en Acadie, voir un excellent article de M. Robert Le Blant, « La Compagnie de la Nouvelle-France et la restitution de l'Acadie (1627-1636) $\gg$ dans la Revue d'Histoire des Colonies (RHC), t. 42, No 146 (1er trimestre 1955) : 69-93. 
détruits. La Compagnie ne voulait sûrement pas desservir sa cause, en rappelant l'appui qu'elle lui avait donné.

D'après leur exposé, la première intervention des Associés en Acadie eut lieu en 1632. Ils envoyèrent alors des vaisseaux de Bretagne et de La Rochelle, sous le commandeur Isaac de Razilly. L'année suivante, la Compagnie expédia encore des rafraîchissements, par «son vaisseau appelé le Saint-Jean », et ces embarquements lui coûtèrent 50,000 écus $(150,000$ livres $)$. Il semble régner ici une équivoque. Car Isaac de Razilly, dans une lettre à Richelieu, ${ }^{6}$ déclare aussi avoir avancé, lui et ses amis, plus de 50,000 écus. S'agit-il de deux sommes différentes ? Cela paraît bien invraisemblable: car les armements d'Acadie auraient alors coûté en deux ans la somme de 300,000 L., autant que les deux flottes envoyées à Québec, et que tout le capital des Cent Associés. Il semble évident que ces deux textes font allusion à la dépense totale de l'entreprise acadienne, à laquelle la Compagnie aurait pris part, en même temps qu'Isaac de Razilly et quelques autres. La Compagnie, à court de fonds après les pertes occasionnées par les Kirke, avait adopté la pratique de recourir à des compagnies particulières, à qui elle accordait une part de la traite, à condition qu'elles participent au financement. On connaît plusieurs de ces sociétés subsidiaires: celle de Bordeaux, celle de Miscou, et les deux de Québec. Une entente à peu près semblable dut régir l'établissement acadien dès 1632 , puisque dans l'armement d'un vaisseau, L'Espérance-en-Dieu, d'Aulnay agit à la fois au nom de la Compagnie et au nom de Razilly. ${ }^{7}$

On peut donc croire que si la Compagnie fournit des vaisseaux, et Richelieu 10,000 L., les Razilly et leurs amis fournirent la majeure partie des fonds nécessaires ; car un arrêté de compte du 20 juin 1632, au moment du premier embarquement, indiquait déjà qu'Isaac de Razilly devait à son frère 53,200 L. ${ }^{8}$ En 1634 intervint un nouvel accord par lequel la Compagnie cédait à

${ }^{6}$ Lettre du 25 juillet 1634. Copie de Margry à la B.N., N.A.F., 9281:60 et $9282: 48$.

7 Archives de la Charente-Maritime, minutes Teuleron, (in Le Blant, loc. cit., 83) et Minutes Tongrelou, liasse 1629-33, acte du 26 nov. 1632 . Voir note 19.

. 
Claude de Razilly, "en considération des grands soins que son frère prenait pour la colonie », les deux forts de La Hève et de Port-Royal, et moitié de la traite pour dix ans. ${ }^{9}$ Cette concession confirme, croyons-nous, la part importante que les Razilly avaient prise jusque là dans la colonie. Autrement, il serait incompréhensible qu'une société, aux trois-quarts ruinée, ait ainsi abandonné une somme de 50,000 écus, deux ans seulement après les avoir investis.

Cette concession signifiait aussi que la Compagnie des Cent Associés s'avouait tacitement incapable, dès 1634, d'assumer ses responsabilités en Acadie, et s'en déchargeait sur des particuliers, comme elle l'avait fait à Québec. Isaac de Razilly et son frère Claude formèrent donc une compagnie privée, où entrèrent Richelieu et quelques autres, et prirent l'entière responsabilité de la colonie acadienne.

Jusqu'au décès du Commandeur, les relations semblent avoir été excellentes entre les Razilly et la compagnie de la NouvelleFrance. C'était d'ailleurs Isaac de Razilly lui-même qui avait suggéré à Richelieu la fondation de cet organisme. Le Factum, comme Champlain et Denys, ne lui décerne que des louanges. Il n'est pas certain que les Razilly aient éprouvé autant de satisfaction de la Compagnie, mais ils n'en soufflèrent mot. Tous deux paraissent avoir été de grands caractères, aux larges vues, très généreux, accommodants avec leurs collaborateurs, et très peu latins, puisqu'on ne les voit tremper dans aucune querelle, mais régler à l'amiable les différends qu'ils ne purent éviter.

Jusqu'à ce moment le Factum a gardé le ton narratif. Mais avec l'entrée en scène d'Aulnay, il prend une allure agressive, et dresse contre lui une longue série de griefs. On lui reproche d'abord de s'être emparé frauduleusement du pouvoir, et d'avoir tout simplement dépossédé la compagnie Razilly. A la mort du Commandeur, écrit-on,

9 Concession du 15 janvier 1634, mentionnée dans un Arrêt du Conseil, A.N., $\mathrm{V}^{6}: 308$, (in Le Blant, loc. cit., 86, n.2) et dans un mémoire des enfants de Latour, Col. C11D, 1:55v. 
« le sieur d'Aunay Charnizay, qui estoit proche de sa personne \& recevoit de luy appointement, se rendit habile à luy succéder, s'empara du Gouvernement, $\&$ se fit le maistre dans lesdits forts et habitations. ... ledit sieur d'Aunay se saisit de tous leurs effects, mania les affaires avec une si estrange oeconomie, que les Associez furent incontinent contraints de luy tout abandonner. 》 p. 4

L'entrée en charge du nouveau commandant présente en effet quelque mystère. Par quelles manœuvres, ou par quel heureux concours de circonstances, ce simple subalterne réussit-il à prendre la succession de son chef, et accaparer en quelques années toutes les affaires de l'Acadie ? Avant de répondre à cette question, il faut d'abord déblayer le sujet, en détruisant une légende notoirement fausse. Le commandeur de Razilly, avant de venir en Nouvelle-France, avait obtenu une commission pour recevoir les places occupées par les Anglais, et un duplicata de cette commission, pour déléguer quelqu'un à sa place, s'il n'y pouvait aller. M. Charles Moreau a prétendu que Razilly, avant de mourir, avait rempli cette commission du nom d'Aulnay, le désignant ainsi comme son successeur. Si l'on se reporte aux sources, ${ }^{10}$ il saute aux yeux que les textes n'autorisent aucunement cette interprétation. La commission en blanc que reçut Razilly ne portait que sur un objectif précis, très limité, la reprise de Port-Royal, comme celle d'Emery de Caen, pour la reprise de Québec. Elle différait absolument de ses lettres de nomination comme lieutenant-général du Roi. ${ }^{11}$ On ne voit nulle part qu'il ait existé un double en blanc de cette nomination. Même s'il eût voulu transmettre ses pouvoirs de gouverneur, Razilly ne le pouvait pas; ce choix dépassait ses attributions. Aucun gouverneur n'a joui du privilège de désigner son successeur. La haute fonction de lieutenant-du-Roi, par sa nature même, relevait direc-

10 Copies anciennes aux Affaires étrangères, Mém. et Doc., Amérique, 4:112 \& seq. et dans Col. C11D, 1:49 et 110. Reproduites dans Coll. de Manuscrits ..., 1:110 et dans plusieurs autres ouvrages.

11 Le texte intégral de ces lettres n'est pas connu. Le marquis de Razilly, dans la Généalogie de la famille de Razilly, Laval 1906, en indique la date: 20 avril 1632. M. Léon Deschamps, dans sa thèse latine, $D e$ Raziliis, Paris 1898, dit (p. 70) en avoir vu une copie dans les papiers de la famille, collationnée par les notaires parisiens Demas et Bergeon (sic). 
tement du souverain. La compagnie seule jouissait, par privilège, du droit de présentation.

Tout au plus, Razilly mourant pouvait-il confier l'administration temporaire de la colonie à l'un de ses nombreux auxiliaires. Trois au moins de ces collaborateurs immédiats, en plus d'Aulnay, nous sont connus: Nicolas Denys, Du Breuil et Poincy. Or un acte, dressé après la mort de Razilly et accompagnant l'inventaire de ses biens, ${ }^{12}$ nous montre que l'homme désigné par Razilly, comme commandant provisoire, ne fut pas d'Aulnay mais le sieur de Poincy. ${ }^{13}$ La légende mise en circulation par Charles Moreau, au sujet de la prétendue nomination d'Aulnay, in-extremis, est donc un bel exemple d'interprétation tendancieuse, absolument erronée, et répétée ensuite sans vérification.

Comment d'Aulnay obtint-il donc le commandement ? Les accusations du Factum baignent dans le vague, et les documents précis, contrats ou procurations, qui pourraient nous éclairer, manquent totalement. Nous possédons cependant quelques données, qui nous permettent de conjecturer comment les événements ont dû se passer. A la mort du Commandeur de Razilly, Claude, son frère cadet, devenait, à côté de Latour, le principal propriétaire de l'Acadie: d'abord comme créancier et héritier de son frère; puis comme principal actionnaire de la compagnie particulière, dont il détenait ou acquit quatre parts sur six; enfin parce qu'il possédait en son nom personnel les titres de concession de La Hève et Port-Royal. Le rôle de ce personnage est peu connu, mais il s'occupa de l'Acadie presque aussi activement que son frère. Il lui avait avancé de fortes sommes, maniait ses affaires en France, organisa la compagnie Razilly-Condonnier. En 1635 , le véritable successeur d'Isaac de Razilly, le fondé de pouvoirs, financièrement et juridiquement, le véritable chef de la colonie, ce fut donc Claude de Launay-Razilly, bien que son autorité n'ait jamais été sanctionnée par une nomination officielle du Roi.

12 Signalé par M. le Blant, loc. cit., 89.

13 Ce personnage n'est pas clairement identifié. Il s'agit probablement du Sieur de Poincy, associé à la part de Condonnier dans la compagnie de Razilly. Un mémoire postérieur des fils Le Borgne lui donne le titre de commandeur; Col. C11D, 1:68. 
Retenu en France par sa famille et sa carrière dans la marine, Claude de Razilly connaissait depuis longtemps Charles d'Aulnay, son parent. Il est tout naturel qu'il ait songé à lui confier la conduite de ses affaires en Acadie. Il vint lui-même au moins deux fois au Canada, mais semble avoir laissé en Acadie toute initiative à d'Aulnay, et même l'honneur de la lieutenance du Roi. Lui-même s'occupait des affaires de la société en France; il fit personnellement des engagements et arma des vaisseaux. Mais le plus souvent il agissait par son représentant à La Rochelle, Nicolas Denys.

D'Aulnay usa-t-il, comme l'insinue le Factum, d'intrigues, de pressions indues ou de procédés frauduleux pour obtenir la direction de l'entreprise ? Rien ne le prouve. Il hésita au contraire plusieurs années, semble-t-il, avant de s'engager définitivement en Acadie. De 1635 à 1640, il n'apparaît dans toutes les transactions commerciales, que comme lieutenant de Razilly, confirmé il est vrai par le Roi en 1638, mais sans aucune propriété au pays, sauf peut-être quelque domaine à Port-Royal. Les premiers droits qu'il acquit dans la compagnie de Razilly durent lui venir par son mariage. Il avait en effet épousé Jeanne Motin, fille d'un actionnaire dans la compagnie de Razilly. Cette jeune fille était venue au Cap-Breton en 1636, avec ses deux sœurs et son beau-frère, Nicolas Le Creux, sieur Du Breuil. Le pays lui plut, puisqu'elle accepta d'y demeurer. Leur mariage dut avoir lieu à Port-Royal, et l'épousée lui apporta probablement en dot les intérêts que détenait son père. Ce n'est que plusieurs années après, nous dit-il lui-même sans beaucoup de précision, qu'il décida de se lancer à fond dans l'entreprise acadienne.

Vers cette époque, la compagnie de Razilly se trouvait en assez mauvaise posture, par suite surtout de la sauvage concurrence qui s'amorçait en Acadie, entre Latour, soutenu par les Bostonnais, et d'Aulnay. A la suite de l'attaque de Latour contre Port-Royal, en 1639, Razilly tente un effort l'année suivante pour secourir son lieutenant, en lui envoyant 25 hommes. Mais les finances de la compagnie sont en mauvais état, et les salaires de l'année précédente n'ont pas tous été payés. ${ }^{14}$. Au cours de cette 27 mars.

14 Archives de la Charente-Maritime, Minutes Teuleron, liasse 1640, 
année surviennent encore de nouvelles difficultés. La saison de pêche apporte peu de profit et, à l'automne, Razilly, pour rembourser les emprunts, doit vendre son vaisseau, le Saint-Jean, et le prix suffit à peine à solder ses dettes. ${ }^{15}$ Par ailleurs, Latour attaque ouvertement d'Aulnay; au cours du combat, le pilote Jamin est tué. Sa veuve intente une action en dommages contre d'Aulnay et fait saisir un navire, le Saint-François, avec les pelleteries qu'il apportait en France. Razilly fait intervenir la Compagnie de Nouvelle-France, et réussit à récupérer ses pelleteries. ${ }^{16}$ On conçoit cependant que cette guérilla et ces procédures devaient causer beaucoup de dépenses et paralyser complètement le commerce.

On ne peut blâmer d'Aulnay, en cette occurrence, puisqu'il ne faisait que se défendre contre les agressions de Latour. Se plaignit-il de ne pas recevoir l'encouragement nécessaire, ou songea-t-il à quitter le service de la compagnie ? Toujours est-il que le vaisseau qui repassait en Acadie, au printemps de 1641, lui apportait un acte notarié, par lequel Razilly, « désirant le favorablement traiter et obliger à embrasser les intérêts et avantages des associés » lui accordait gratuitement une part dans la compagnie. ${ }^{17} \mathrm{Si}$ les termes de cet acte contiennent un léger reproche, il n'est qu'indirect et voilé, à peine exprimé. D'Aulnay y paraît bien devenu un homme indispensable, dont on veut s'assurer l'entière collaboration, par des largesses.

A l'automne de la même année, d'Aulnay passe en France, et peu après intervient l'importante transaction qui fait de lui le maître de l'Acadie. Le 16 janvier 1642, Claude de Razilly lui vend ses quatre parts dans la compagnie particulière, moyennant la somme de $14,000 \mathrm{~L}$. payables en sept versements annuels. ${ }^{18}$

15 Ibid. id. registre $1640: 91,23$ novembre.

16 Ibid. B.190, Amirauté de La Rochelle, 1639. B.N., N.A.F., 9281-84.

17 Acte du 27 février 1641. B.N., Pièces originales, dossier Menou, p. 53. Reproduit par Fauconneau-Dufresne, Rapport sur les Preuves de l'histoire de la maison de Menou, 37, et par Moreau dans Le Cabinet historique, 13 (1867) : 185.

18 Ce contrat, passé à Paris le 16 janvier 1642, devant Lemercier et Chapelain, nous est connu par un Arrêt du Conseil d'Etat, A.N., No 5E, 1688, cité par l'abbé Couillard-Després, (Charles de S. Etienne de Latour et son temps, 300, 303), par le testament d'Aulnay et par un mémoire des enfants Latour; C11D, I: 55v. L. Deschamps (De Raziliis, 79) en mentionne une copie dans les papiers de la famille Razilly. 
Le 19 février suivant, «en considération et reconnaissance des soigns et travaux de messire Charles de Menou ... et en faveur de l'amitié et parenté qui est entre eux 》 il lui donne encore 4,000 L. à prendre sur la succession de son frère, pour acheter la seigneurie de Sainte-Croix. ${ }^{19}$

De ces divers marchés résultait pour Razilly une perte considérable, puisqu'il cédait au prix dérisoire de $14,000 \mathrm{~L}$. des établissements qui lui en avaient coûté au moins 50,000. Ces offres alléchantes, à moins qu'on ne considère Razilly comme une dupe, trahissaient son grand désir, et même son anxiété de se retirer des affaires d'Acadie. Jusque là, il avait avancé des sommes considérables dans la colonisation et le commerce de Nouvelle-France. Depuis les débuts, il avait participé aux activités de la Compagnie des Cent Associés, et fait partie des deux compagnies particulières du Saint-Laurent. La première avait rapporté des profits, mais la seconde éprouva de lourdes pertes. ${ }^{20}$ En Acadie, Latour et les Bostonnais prenaient une attitude aggressive. Pour leur tenir tête, il fallait des vaisseaux, des soldats, des canons, de l'argent. Tout reposait sur les épaules de Razilly, et sa fortune était alors gravement compromise. Épris de grandes idées coloniales, et soutenu par Richelieu, il s'était engagé à fond dans les affaires d'Amérique, mais les résultats matériels n'avaient pas répondu à son attente. En 1642, à demiruiné et assailli par les mauvaises nouvelles qui ne cessaient de lui arriver d'Acadie, on comprend facilement qu'il ait commencé d'en avoir assez, et voulu se décharger, à tout prix, de cette responsabilité.

D'autre part, on peut juger que le petit lieutenant, parti de rien en 1635, sans un sou vaillant dans la colonie, a bien manœuvré, puisqu'après 8 ans il domine l'entreprise. Mais il assumait en même temps d'énormes responsabilités; telles justement que la Compagnie s'en était déchargée sur les épaules des Razilly, et que Claude de Razilly lui-même refusait de les porter plus longtemps. Les deux parties possédaient assez d'expérience

19 Copie de Margry à la B.N., N.A.F., 9281:89. Reproduite par Fauconneau-Dufresne, loc. cit., 41.

${ }^{20} \mathrm{La}$ Roncière, Histoire de la Marine française, 4:460. 
et de réalisme pour ne pas se leurrer d'illusions, et savoir que la propriété d'une telle entreprise ne comportait pas seulement les bénéfices du commerce, mais imposait des charges de colonisation et d'administration, des obligations de police onéreuses, et d'inquiétants risques de guerre.

On comprend que la grande compagnie, dont d'Aulnay n'a pas sollicité les faveurs, le considère comme un parvenu. Avouons qu'il en présente tout l'air. Mais il avait travaillé rudement, pour en arriver là. En tout cas, s'il avait employé des procédés malhonnêtes pour parvenir à ce résultat, c'était aux principaux intéressés, à Razilly et ses associés de s'en plaindre. Or, on ne trouve aucune plainte de ce genre. Il semble bien, au contraire, que ce soit Razilly qui ait fait pression sur d'Aulnay, par ses générosités, pour lui faire accepter sa succession. Nicolas Denys, parfaitement au courant des affaires de Razilly, et plutôt antipathique à d'Aulnay, dit simplement qu'il survint entre eux «un accommodement $»^{21}$ Il nous faut donc reconnaître que la première accusation du Factum manque de preuve. Elle peut nous présenter un d'Aulnay habile et ambitieux, mais elle ne réussit pas à le montrer malhonnête. ${ }^{22}$

Le second grief de la Compagnie est beaucoup plus précis et mieux justifié. Elle reproche à d'Aulnay d'avoir lésé ses droits, en se faisant nommer à la charge de lieutenant-général, ou gouverneur, sans passer par elle.

Le droit de présenter les gouverneurs et les officiers supérieurs figurait explicitement dans la charte de la Compagnie, et elle en avait usé jusque là. Dans les nominations de Razilly et de Latour en Acadie, dans celles de Champlain et de ses successeurs à Québec, on a toujours soin d'indiquer leur présentation par la Compagnie. Mieux que cela, plusieurs gouverneurs sortaient de ses rangs.

21 Nicolas Denys, Description..., 100; édition Ganong, 483.

22 Les autres actionnaires, Tardif et Poincy, refusèrent d'avancer de nouveaux fonds, et semblent avoir abandonné leurs parts: L. Deschamps, De Raziliis, 79; Testament d'Aulnay, in Candide de Nant, Pages glorieuses ..., 320; Mémoire des fils Le Borgne, Col., C11D, 1:68. 
En Acadie, après la mort d'Isaac de Razilly, le poste de gouverneur vaqua plusieurs années. D'Aulnay exerça d'abord cette fonction sans en porter le titre. Cette autorité de fait lui fut reconnue, incidemment, une première fois par une lettre de Louis XIII, en 1638. Une confirmation plus formelle lui parvint en 1641, avec un mandat contre Latour. D'Aulnay utilisa toutes les erreurs de son adversaire pour augmenter son crédit et consolider ses positions. Dans ces diverses occasions, ni lui ni ses amis ne consultèrent la Compagnie, mais adressèrent leurs mémoires directement à la Cour.

Bien que d'Aulnay et la Compagnie eussent forcément des relations commerciales, l'hostilité, née avec la prise de Pentagouët en 1635, continuait de s'envenimer. Dans la donation du fief de Sainte-Croix, en 1642, Razilly inscrit une clause révélatrice. Il cède tous ses droits, mais aux risques et périls du sieur de Menou, sans aucune garantie, quoi «que les intéressez en la Compagnie de Nouvelle-France voullussent et pussent contre ladicte concession $\gg .{ }^{22 b}$ Plusieurs indices nous montrent que par ailleurs, dans la lutte d'Aulnay-Latour, la Compagnie sympathisait, au début du moins, avec Latour. Hutchison nous dit ${ }^{23}$ que ce dernier montra au Conseil de Boston une lettre d'un agent de la Compagnie, l'informant des menées d'Aulnay et l'avertissant de bien prendre garde à lui. Quelques années plus tard, en présentant ses défenses au Conseil d'État, Latour demanda «attendu que les associés et directeurs de la Compagnie de Nouvelle-France avaient intérêt à ce que le pays ne tombât pas sous le pouvoir de personnes qui ne leur seraient pas agréables, qu'il fut ordonné que lesdits directeurs et associés seraient assignés en l'instance ». ${ }^{24}$ Latour comptait donc obtenir devant le Conseil l'appui de la Compagnie contre d'Aulnay, mais elle ne bougea pas.

Il fallut, pour la tirer de sa torpeur, les lettres patentes accordées à d'Aulnay en 1647. Cette fois la Compagnie se sentit directement menacée et réagit vivement. Il y avait de quoi. Louis XIII et Richelieu, protecteurs de la Compagnie, étaient morts. La reine régente, Anne d'Autriche, et Mazarin les avaient

22b Voir plus haut note 19.

23 The History of Massachusetts Bay..., (London, 1760), 1:130.

24 Couillard-Després, loc. cit., 348. 
remplacés. D'Aulnay, qui venait de prendre le fort Latour, se donnait à la Cour, appuyé par son père et les Capucins, des airs de sauveur. Il venait de s'endetter de $200,000 \mathrm{~L}$., disait-il, pour. repousser les ennemis de l'État, et réclamait de l'aide. La Reine, dont le trésor était vide, lui promit un bateau, qu'elle n'envoya pas, mais lui fit décerner, en revanche, un beau parchemin. Le motif en valait la peine. D'Aulnay se ruinait pour établir une colonie et défendre l'honneur de la Couronne; ce dévouement valait bien en récompense un petit effort de rhétorique de la part du ministre, et quelques largesses au Nouveau-Monde.

D'Aulnay eut sans doute préféré un vaisseau bien approvisionné, mais il dut quand même éclater de joie à la lecture de ce parchemin. Aucun de nos gouverneurs canadiens ne reçut de nomination ni de privilèges aussi hyperboliques. ${ }^{25}$ L'exagération de leurs termes nous étonne encore. Elle dut estomaquer les Cent Associés. Après un rappel fortement exagéré des services rendus par d'Aulnay, on lui concède tout le territoire acadien, depuis le Saint-Laurent jusqu'à la Virginie, et le privilège exclusif de la traite, avec amende de $30,000 \mathrm{~L}$. aux transgresseurs. Il obtient pouvoir d'y commander par terre et par mer, de nommer les officiers, de faire la paix et la guerre, de se réserver les terres de son choix et de concéder les autres à son bon plaisir, avec les titres, honneurs, droits, grâces et privilèges qu'il voudra; de construire villes, forts, havres ; enfin généralement, dit la Reine, «tout ce que nous pourrions faire si nous y étions en personne, lui donnant à cette fin tout pouvoir, autorité, commission et mandement spécial ». Voilà une nomination princière, une véritable délégation de pouvoirs vice-royaux.

On avait seulement oublié l'existence d'une compagnie, à qui Louis XIII, vingt ans auparavant, avait concédé des droits de souveraineté à perpétuité, sur les mêmes territoires; que ces droits n'avaient pas été révoqués, et que le territoire du Golfe, en particulier, lui appartenait encore en propre et qu'elle l'exploitait effectivement. Les lettres patentes de 1647 entraient donc en

25 Acte de février 1647. Copies anciennes aux Affaires étrangères, Corr. Angleterre 55 supplément: 49 et dans Col. C11D, 10 (non paginé). Reproduit dans les Mémoires des Commissaires, 2:281 et dans Fauconneau-Dufresne, loc. cit., 52-55. 
contradiction flagrante avec la charte de la Compagnie, et violaient catégoriquement ses privilèges. Cet empiètement survenait juste au moment où cette compagnie, réduite à 69 membres, venait d'abandonner (1645) ses droits de traite sur le Saint-Laurent à la Compagnie des Habitants. Comme elle avait déjà concédé la péninsule acadienne, le Cap-Breton et la rivière Saint-Jean, il ne lui restait plus que les rives du Golfe, pour essayer de rembourser, par la traite, un déficit de 400,000 L. L'extension des pouvoirs d'Aulnay non seulement méprisait son privilège de nomination, mais lui causait un grave préjudice financier. La Compagnie avait donc là un grief très sérieux, appuyé sur un solide fondement juridique. Il est probable que, si elle eut voulu pousser sa cause, elle eût obtenu, sinon la cassation de cette nomination irrégulière, au moins un rajustement de ses conditions.

Un cas identique s'était présenté en 1628 , précisément lors de la création de la Compagnie des Cent Associés. Bien que Richelieu eût alors formellement révoqué la compagnie De Caen, pour la bonne raison qu'elle n'avait pas rempli ses obligations, celle-ci n'en obtint pas moins, en compensation, une année de traite et $60,000 \mathrm{~L}$. d'indemnité.

La Compagnie avait d'autant plus raison de se plaindre, que d'Aulnay n'entendait pas s'en tenir à une jouissance platonique de ses pouvoirs, mais prétendait les mettre rigoureusement en exécution. Il avait même déjà commencé depuis longtemps. Le Factum énumère plusieurs actes de violence commis par lui. En 1646, il s'empara, paraît-il, de quelques barques de la Compagnie qui trafiquaient avec les Sauvages, dans le Golfe Saint-Laurent. Les fourrures pillées furent saisies à La Rochelle, par la Compagnie, sur une ordonnance de la cour d'Amirauté. Mais d'Aulnay obtint levée de cette saisie, par un arrêt du Conseil. Nous ne possédons aucun détail supplémentaire sur cette affaire.

En septembre 1647, fort de ses lettres-patentes, d'Aulnay se serait aussi emparé des forts du Cap-Breton et de Miscou, en chassant les colons et les missionnaires. Il s'agit évidemment de la prise du fort Saint-Pierre, contre Jacques Maillet et René 
Guignard, représentants de Louis Guignard, dont le procèsverbal a été conservé. ${ }^{26}$ Quant à la prise de Miscou, Nicolas Denys, qui y commandait pour une compagnie particulière, nous en parle avec aigreur dans son livre et dans ses lettres. D'Aulnay dressa un inventaire des marchandises et promit d'en rembourser le prix, mais il mourut sans l'avoir payé. ${ }^{27}$ Pour compléter ces hautsfaits, la Compagnie aurait pu ajouter l'incendie du fort SaintLouis, au Cap Sable, en 1641, que le Roi lui avait donné instruction de mettre en mains de "personnes fidèles », et la destruction du Fort Latour, en 1645, à la rivière Saint-Jean.

Ces brutalités résultaient de la mise en vigueur de ses diverses commissions. Mais les concessionnaires évincés possédaient eux aussi des commissions régulières de la Compagnie. D'Aulnay pouvait bien se donner des apparences de justicier à l'égard de Latour, qu'il avait d'ailleurs contribué à pousser du côté anglais. Mais il ne pouvait rien reprocher à Denys, pas plus qu'à Guignard. Ces agissements nous inspirent des doutes sur la loyauté de leur auteur. La saisie de navires surpris à pratiquer une traite illicite, dans une région concédée en monopole, était régulière et pratiquée par tous. Mais d'Aulnay, en étendant son domaine au Cap-Breton et dans le Golfe, devait fort bien savoir qu'il empiétait sur un territoire déjà concédé. Même protégées par une commission royale, ces dépossessions ressemblent fort à des procédés de pirate.

On peut invoquer en faveur d'Aulnay les charges très lourdes qu'il devait supporter pour entretenir la colonie et ses garnisons. Sans doute ! Mais cette raison ne l'excusait pas de violer les droits reconnus de ses concurrents. L'appréciation de ces actes, au point de vue de la justice, relevait des tribunaux. Mais l'aspect politique de la question, et l'intervention de la Cour, brouillaient les choses. La Compagnie pouvait bien réclamer une réparation en justice, mais la décision du tribunal risquait, encore une fois, d'être renversée par un arrêt royal. Il fallait au préalable décider la question de droit, à la source. C'est pourquoi la Compagnie s'adressait-elle aussi à la Cour.

\footnotetext{
${ }^{26}$ Col. C11D, 1:81. Voir article de M. Delafosse dans cette revue, vol. 4, No 4 (mars 1951) : 475 et 490 .

27 Nicolas Denys, Description ..., 192; édition Ganong, 68, 203, 499.
} 
Enfin, dernier grief, la Compagnie reproche à d'Aulnay de n'avoir rien fait, ni pour la colonisation, ni pour la conversion des sauvages. Cette accusation était la plus apte à influencer la Cour. Nicolas Denys formulera plus tard contre d'Aulnay le même reproche :

"Tous ces habitants (de Port-Royal), dit-il, sont ceux que le commandeur de Razilly avait fait venir de France à La Haive, qui depuis ce temps ont bien multiplié... (Le commandeur) ne souhaitait que de faire connaître la bonté du pays et de le faire peupler. (D'Aulnay) tout au contraire, appréhendait qu'il s'habitast; et aussi n'y a-t-il fait passer personne $\gg .{ }^{28}$

Cette accusation pose le difficile problème des origines du peuplement acadien, et de la part qu'y prirent Razilly et d'Aulnay. La Gazette de Renaudot parle de « 300 hommes d'élite » amenés par Razilly en 1632. Ce chiffre devait comprendre les matelots, les soldats et les engagés, mais peu de familles. Denys nous dit que La Hève ne comptait qu'une quarantaine d'habitants à l'époque de Razilly, et le premier enfant français de la colonie naquit seulement en 1636. La compagnie de Razilly dut envoyer d'autres renforts, mais nous ne connaissons, à part quelques engagements particuliers, que le contingent de 76 personnes conduit par Dubreuil en 1636, et celui de 25 hommes et 5 femmes, envoyé en $1640 .^{29}$

Comme toujours, une faible partie seulement de ces recrues se fixa au pays. Sur 9 familles, par exemple, arrivées en 1636, 4 seulement demeurèrent en Acadie. Et de 54 laboureurs, charpentiers, sauniers, à peine 5 ou 6 s'y établirent. L'examen des listes d'engagés qui nous sont connues confirme ces constatations. Le chiffre qu'il nous intéresse donc surtout de connaître, c'est de savoir combien de colons stables Razilly et d'Aulnay réussirent à fixer au pays.

28 Id. ibid., 54 et 101 ; édition Ganong, 151, 474, 483.

29 Archives de la Charente-Maritime, Série B, prov. 75, Rôle du SaintJean 1636, reproduit et analysé par le R.P. Archange Godbout, dans les Mémoires de la Société généalogique canadienne-française, vol. 1 . No 1 (janvier 1944) : 19-30. Nous ne possédons aucune liste de la recrue de 1640; voir minutes Teuleron, registre $1640,36 \mathrm{v}$. 
Le Père Pacifique de Provins, préfet des missions capucines, nous fournit à ce propos une indication précieuse. Ses lettres nous apprennent qu'en 1641, juste au moment où Claude de Razilly se retira et d'Aulnay lui succéda, l'Acadie entière comptait, dans ses quatre postes, 120 hommes et 40 soldats..$^{30}$ A peine deux ou trois ans plus tard, un mémoire d'Aulnay nous dit qu'il avait 400 bouches à nourrir, dont 200 soldats, laboureurs et artisans. $^{31}$ Ce chiffre, probablement exagéré, correspond de plus à une époque de pointe, où il entretenait un fort contingent de soldats, pour sa lutte contre Latour. Si l'on se reporte, par ailleurs, au recensement de 1671, vingt ans après la mort d'Aulnay, Port-Royal ne comptait alors que 359 âmes, et l'Acadie entière, environ 400. La conquête anglaise de 1654 avait dû provoquer le départ de plusieurs engagés, mais les naissances avaient dû maintenir le chiffre de la population, et même l'augmenter. On peut donc en déduire, croyons-nous, qu'à la mort d'Aulnay, la population stable de toute l'Acadie ne devait guère dépasser 300 personnes, soit 45 ou 50 familles. Si l'on soustrait de ce chiffre au moins 120 habitants amenés par les Razilly, et l'accroissement normal de ces familles, l'effort colonisateur d'Aulnay ne paraît pas aussi prépondérant que bon nombre d'historiens l'ont prétendu. Le mémoire de 1643, écrit sous sa dictée, sinon par lui-même, affirme qu'il a fait passer « 20 ménages français ». Michel Boudrot, recueillant en 1687 la déposition des anciens du pays, dit simplement que d'Aulnay amena de France «plusieurs familles », ce qui n'est pas très fort. Rameau estime qu'il en établit une vingtaine. ${ }^{32}$ Ce chiffre, tout bien pesé, nous paraît un maximum.

D'Aulnay et ses amis, en leurs mémoires, ont tenté de s'arroger les mérites de leurs prédécesseurs, les Razilly, et veulent donner l'impression qu'il a tout créé en Acadie. Ses ennemis, Latour, Denys, la Compagnie, prétendent au contraire qu'il n'apporta rien. En vérité, Isaac de Razilly avait posé des bases solides, sur lesquelles d'Aulnay put ensuite continuer l'édifice. Lequel

${ }^{30}$ Lettres du 7 nov. et du 12 déc. 1641, à la Propagande. Citées par le R.P. Candide de Nant, loc. cit., 213, note 2.

31 B.N., N.A.F., 9281: 104-105.

32 E. Rameau de Saint-Père, Une colonie féodale en Amérique, 1: 110. 
accomplit davantage ? La pénurie de documents empêche de le préciser exactement. Cela importe d'ailleurs assez peu. Il suffit que d'Aulnay ait maintenu, malgré les difficultés, et développé, même petitement, la colonie, pour qu'il n'ait pas démérité. En tout cas, proportion gardée, d'Aulnay avait sûrement accompli dans son secteur, autant que la Compagnie dans un domaine plus grand. Et la petite population acadienne de 1650 était assez bien enracinée au pays, puisqu'elle survécut, peu après, à une occupation anglaise de 15 ans.

Quant à la conversion des Sauvages, le Factum prétend que, du côté d'Aulnay, «il n'y a pas deux familles de converties », tandis qu'au Saint-Laurent «il y a plus de mille familles sauvages baptisées et chrétiennes ». On sait que d'Aulnay collabora étroitement avec les Capucins, et administrait la part que leur avait léguée Richelieu, pour l'entretien du séminaire. Après sa mort, le Père Ignace, son confesseur, adressait à un ami un témoignage louangeur. ${ }^{33}$ Tous les Capucins cependant ne s'accordaient pas avec d'Aulnay, et quatre d'entre eux avaient été rappelés en France en 1641 «ob justas causas », c'est-à-dire vraisemblablement parce qu'ils ne s'entendaient pas avec le gouverneur. S'il faut en croire Latour, l'un d'eux, le Père Vincent de Paris «a fait son possible pour être ouy contre lui au Conseil, et pour prouver son hypocrisie ». Le Père Pacifique de Provins, préfet des missions capucines en Acadie, l'appelle un «mauvais gouverneur » et l'accuse «d'avoir mangé et détourné à son profit la somme de vingt mille francs laissée par le cardinal Richelieu pour les missions et l'entretien des petits sauvages ». Les Récollets, de leur côté, témoignent contre lui en 1642, et réclament une indemnité pour leurs pertes à la mission du Cap Sable, détruite avec le fort de Latour, l'année précédente. ${ }^{34} \mathrm{Au}$ CapBreton et à Miscou, le Factum prétend que d'Aulnay en 1647 «a chassé les habitants, et jusques aux Ecclésiastiques qui rési-

33 Cette lettre du Père Ignace, véritable panégyrique, est reproduite sans indication de source dans Coll. de Manuscrits, 1:136, et par le R.P. Candide de Nant, loc. cit., 325.

34 Ces divers témoignages contemporains, contre d'Aulnay, sont rapportés par l'abbé Couillard-Després, loc. cit., 21, 29, 304-305, 311, 359. 
doient ausdits lieux pour l'instruction des pauvres Sauvages ». (p. 5) Que penser de tout cela ?

Il est possible que le changement d'allégeance ait éloigné momentanément les Jésuites de Miscou et du Cap-Breton. En effet la Compagnie de Miscou et surtout l'un de ses membres, l'abbé de la Magdeleine, soutenaient puissamment les Jésuites à Miscou et leur avaient même fait construire une habitation à Nepisiguit et des maisons pour les Sauvages convertis. L'évincement de la compagnie dut tarir ces générosités. Les lettres et Relations de 1647 ne disent cependant pas un mot de la prise du fort par d'Aulnay. Mais, est-ce par simple coïncidence ou par suite de cette spoliation, aucun des trois missionnaires Jésuites qui desservaient la côte, de Gaspé à Canso, ne s'y trouve plus à l'automne de 1647.35

Il est évident que d'Aulnay a pu commettre des fautes, mais ses bonnes relations avec la majorité des Capucins, la confiance que lui témoignèrent les supérieurs de cet ordre, la lettre du Père Ignace et son propre testament nous le montrent comme un chrétien sincère. Oh ! nous n'irons pas jusqu'à le canoniser, et à le couronner "saint de l'Acadie», comme l'a proclamé un peu trop hâtivement un de ses biographes. ${ }^{36}$ Mais il valait sûrement la plupart des chrétiens de son temps. Les soucis de l'administration, du commerce et de la guerre l'empêchèrent peut-être de collaborer avec les Capucins autant qu'ils collaborèrent avec lui, mais il n'entrava pas leur travail. Quand la rivalité commerciale l'amena à s'ingérer dans les établissements de ses concurrents, cette action put nuire indirectement aux missions dans ces lieux. Mais ce ne fut qu'accidentel et temporaire. Car, dès 1649 on retrouve les Jésuites à Miscou, ${ }^{37}$ et les Capucins assurèrent la relève au Cap-Breton et sur les côtes du Golfe, de Nepisiguit à Chedabouctou. ${ }^{38}$ Quant aux discussions de la Compagnie, à savoir

35 Relation de 1647. Thwaites, Jesuit Relations ..., 32:52 et $33: 34$.

36 Pierre de la Vaissière, «Le Saint de l'Acadie: Charles de Menou d'Aulnay ». RHC, t. 12 (1924) : 473-494. Article basé uniquement sur le testament d'Aulnay, dont le texte est reproduit.

37 Thwaites, loc. cit., $34: 57$ et $43: 22$.

38 Voir la lettre latine du Père Ignace, aux Archives de la Propagande, reproduite par le R.P. Candide de Nant, loc. cit., 305-311 et reproduite aussi avec traduction dans $R A C .1905$, vol. 1, appendice $\mathrm{H}$. 
qui, d'elle ou de son adversaire, avait accompli davantage pour la conversion des indigènes, le ton même du Factum nous indique que cet argument ne visait qu'à plaire à la Reine-Mère, très dévote. Quand les partis se mettent à discuter des services qu'ils rendent à la religion, on devrait leur conseiller plutôt de rivaliser en désintéressement et en modestie.

Quelles furent les suites de ce Factum? La Compagnie crutelle imprudent d'intenter une poursuite qui aurait risqué d'attirer l'attention sur sa propre insuffisance à remplir ses obligations coloniales? Ou jugea-t-elle la situation d'Aulnay trop forte pour l'attaquer? Il semble qu'elle ne poussa pas très loin ses protestations, et que cette pièce ne servit qu'à sonder le terrain. La mort d'Aulnay, en 1650, vint résoudre ces difficultés. Ou plutôt les remplacer par d'autres; car, dès l'année suivante, la Cour passait encore une fois par-dessus la tête de la Compagnie, en accordant directement d'autres lettres-patentes, et cette fois, nouvelle incohérence, à Latour, l'ancien adversaire acharné d'Aulnay. Latour n'avait peut-être pas tort de dire qu'à cette époque, à la Cour de France, il suffisait de 20 écus pour acheter un arrêt. ${ }^{39}$

Mais la Compagnie n'abandonnait pas ses droits puisque, cinq ans plus tard, elle obtiendra l'annulation des lettres de Latour, sous prétexte qu'elle ne l'avait pas présenté, et lui substituera Le Borgne. Par la suite, elle obtint le respect au moins théorique de ses droits, car, dans la nomination des gouverneurs suivants, on invoquera la présentation des candidats par la Compagnie.

En somme, que nous apprend ce Factum? Ajoute-t-il simplement un chapitre de plus à l'épopée de nos chicanes nationales? Ou bien, nous apporte-t-il des détails et des aspects nouveaux? Voici, à notre avis, ce que nous en pouvons retenir. Il confirme d'abord les témoignages de Champlain et Denys sur le comman-

39 Rapporté dans une lettre d'Aulnay au gouverneur de Boston, 31 mars 1645, reproduite par Fauconneau-Dufresne, loc. cit., 47. 
deur Isaac de Razilly, et met un peu mieux en lumière le rôle de son frère, Claude de Launay-Razilly.

Sur la Compagnie des Cent Associés, il nous montre, sans le vouloir, que son rôle en Acadie fut relativement faible. Dès 1631, elle avait les reins brisés, et se libéra de ses obligations, en les confiant à des compagnies subsidiaires. Elle eut beau s'évertuer à maintenir ses pouvoirs et réclamer ses droits, sa longue agonie ne fut par la suite qu'une série d'expédients et de démissions.

On ne peut s'empêcher, non plus, de former quelques réflexions sur le gouvernement d'Anne d'Autriche et de Mazarin. Quand on voit cette administration si mal soutenir l'effort de ses colonisateurs, se contredire elle-même et laisser ses sujets se chamailler entre eux, on doit en conclure que ce fut un très piteux régime, au moins pour les colonies.

Contre d'Aulnay, ce Factum constitue un puissant réquisitoire. Malgré ses exagérations et quelques faussetés, il confirme et donne un certain crédit aux accusations venues d'autres sources. Que Denys évincé en veuille à d'Aulnay, cela se comprend. Si Latour l'accuse, nous écoutons ces dénonciations comme celles d'un adversaire partial. Mais quand de graves et puissants personnages, comme les Directeurs de la Compagnie de NouvelleFrance, entrent dans le débat, et que des Récollets et quelques Capucins y joignent leur mot, ces témoignages forment un impressionnant faisceau, et il faut bien en tenir compte, au moins sur certains points.

On ne peut nier qu'il nous apparaît alors comme un terrible ambitieux. Le Factum ne dit pas un mot de la longue querelle entre d'Aulnay et Latour. Mais les disputes d'Aulnay avec la Compagnie, et avec ses autres concurrents, forment des épisodes parallèles et manifestent le même esprit d'avidité. Sans aucun doute d'Aulnay avait conscience de travailler pour le bien de la colonie, et nous voulons croire à la droiture de ses intentions. Mais il eut tort de vouloir identifier la colonie avec son gouverne-ment et sa personne, et de vouloir jalousement tirer à lui toute l'autorité et tout le commerce, au lieu de collaborer avec ses partenaires. Son ambition, comme une pieuvre, voulut étouffer de 
ses tentacules, d'abord Denys, puis Latour, puis Guignard, puis encore Denys à Miscou, puis la Compagnie de la Nouvelle-France elle-même. S'il avait employé à son œuvre constructive le temps, l'argent, les hommes, les procédures qu'il engagea dans ces luttes, la colonie y eût sûrement gagné.

Est-ce à dire que son œuvre fut entièrement négative? Non pas. En toute équité et objectivité, il faut reconnaître que ni les erreurs d'Aulnay, ni ses adversaires ne lui enlèvent ses grands mérites. Elles le présentent plutôt sous un jour différent, sous un double éclairage, dirions-nous. A un certain point de vue, uniquement psychologique, on peut même dire qu'il y gagne. Car, pour parvenir, en partant de rien, à s'emparer d'une puissante entreprise, à maintenir cinq forts et une petite flotte, à entretenir 400 personnes, à faire en même temps de l'agriculture, du commerce, de la guerre et de la diplomatie, à obtenir de ses bailleurs de fonds 200,000 L. d'avances à lui seul, c'est-à-dire une somme égale aux deux tiers de tout le capital des Cent Associés, et à porter échec même à cette puissante compagnie, il fallait un cerveau puissant et méthodique, un plan bien arrêté et, pour le réaliser, une habileté consommée, en plus d'une énergie inlassable. En fait, il triompha de tous ses adversaires, et ses succès un peu machiavéliques lui donnent vraiment grande taille.

On ne peut nier qu'il accomplit aussi beaucoup pour l'Acadie. Même en restituant aux Razilly la juste part qui leur revient, et même si d'Aulnay n'accrut pas très considérablement la colonie, il la maintint et l'affermit. Il établit des fermes, endigua des marais, édifia des moulins, construisit des vaisseaux, et traita bien ses censitaires. Il enracina ainsi les colons au pays, et fit de Port-Royal une solide communauté agricole, qui put subsister par elle-même sous l'occupation anglaise. Abandonné à ses seules ressources, il sut aussi négocier et maintenir la paix avec ses voisins de Nouvelle-Angleterre. Par son attitude énergique, sa réputation de chef prudent et courageux, ses forts bien munis de - canons et de soldats, il imposa le respect et soutint l'honneur de la France. S'il avait vécu en 1654, Sedgewick n'eût probablement pas osé l'attaquer, ou n'eût pas, en tout cas, remporté un succès si facile. 
Le Factum ne peut détruire ces aspects positifs de la carrière d'Aulnay. Mais, en un certain sens, il les complète, ou les équilibre. De l'ensemble des documents, nous voyons surgir sa figure contrastée. De fortes ombres, certes, s'y dessinent, mais elles rendent cette figure plus humaine, plus vivante, plus dramatique. Et nous l'aimons tout autant. Aux fausses couleurs des panégyristes, nous préférons la crudité du portrait vrai.

D'Aulnay nous apparaît ainsi très digne de figurer dans cette galerie d'hommes et de femmes exceptionnels qui marquèrent les origines de l'Acadie et du Canada. Malgré leurs défauts et leur rivalités, ces hommes eurent tous un trait commun, ils furent grands, au moins par quelque côté de leur caractère. Ils dépassaient le commun. Et c'est peut-être pour cette raison qu'ils vinrent en Amérique, afin d'y trouver un champ d'action et des horizons à leur taille.

Un sentiment de tristesse nous saisit cependant en étudiant leurs vies; c'est qu'ils aient trop souvent usé leurs forces à se combattre les uns les autres. Mais si nous regardons nos divisions actuelles en plusieurs domaines, on ne peut vraiment pas leur jeter la pierre, et il nous faut avouer que nous avons bien peu profité des leçons de l'histoire.

RENÉ BAUDRY, c.S.c., Université Saint-Joseph, N.-B. 